\title{
Swarming and swirling in self-propelled polar granular rods
}

\author{
Arshad Kudrolli ${ }^{\dagger}$, Geoffroy Lumay ${ }^{\dagger, \ddagger}$, Dmitri Volfson*, Lev S. Tsimring* \\ $\dagger$ Department of Physics, Clark University, Worcester, MA 01610 \\ $\ddagger G R A S P$, Physics Department, University of Liège, B-4000 Liège, Belgium \\ * Institute for Nonlinear Science, University of California, San Diego, La Jolla, CA 92093
}

\begin{abstract}
Using experiments with anisotropic vibrated rods and quasi-2D numerical simulations, we show that shape plays an important role in the collective dynamics of self-propelled (SP) particles. We demonstrate that SP rods exhibit local ordering, aggregation at the side walls, and clustering absent in round SP particles. Furthermore, we find that at sufficiently strong excitation SP rods engage in a persistent swirling motion in which the velocity is strongly correlated with particle orientation.
\end{abstract}

PACS numbers: 45.70.Qj, 05.65.+b

Large-scale structures emerge spontaneously in systems of interacting SP biological objects such as flocks of birds, schools of fish, amoebae colonies, as well as in multi-robot swarms [1, 2]. Chemotaxis and field gradients can lead to non-equilibrium aggregation 3], and hydrodynamic interactions can cause vortices [4]. Such observations prompted a discrete-time, discrete-element model [5] where SP point particles ("boids") align their velocities with the average velocity of other particles within a certain fixed-size neighborhood. This model predicts a spontaneous phase transition to a long-range ordered state as the noise (temperature) of the system is reduced below a critical value, however the exact nature of the transition is still a matter of debate $[6]$. Continuum hydrodynamic-type field models for a population of SP particles have been derived either general symmetry arguments [7] or directly from microscopic interaction rules [8]. These models allowed for detailed predictions of the correlation properties within the ordered state. However, both these models did not explicitly take into account the finite size and shape of interacting particles. On the other hand, there have been rapid advances in the theory of "active nematics", or populations of inelastically interacting rods, both polar [9, 10] and apolar [11, 12]. These models predict onset of a nematic order when the coupling strength of particle density becomes sufficiently high, furthermore, clustering of apolar rods can lead to giant density fluctuations. Clustering of polar rods was recently found in numerical simulations 13]. On the experimental side, there has also been growing interest in the nonequilibrium dynamics of driven granular rods. Symmetric rods in a vibrated container have been shown to form nematic or tetratic order and under certain conditions exhibit persistent swirling [12, 14, 15], and giant number fluctuations [16]. At higher density, rods begin to bounce on one end and travel in the direction of their tilt due to friction at the contact between the rod and the substrate [17]. Collectively, these rods spontaneously form large scale vortices [18, 19].

Here, we study experimentally and numerically the collective dynamics of vibrated polar granular rods which

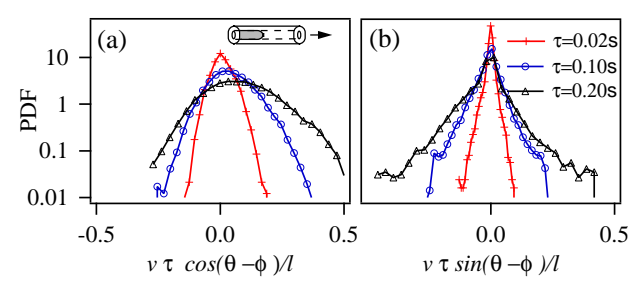

FIG. 1: The distributions of displacements in a time interval $\tau$ (a) parallel, and (b) perpendicular to axis of the polar rod $(\Gamma=2)$. Inset: Schematic of the polar rod. Arrow shows the direction of net motion.

have a non-symmetrical mass distribution but retain their shape symmetry. Such a rod on a vibrated surface moves towards the lighter end. When many such rods are placed inside a vibrated container, for weak excitation they aggregate over time at the boundaries. When the magnitude of excitation is increased, aggregation at the boundaries is reduced, and coherent structures are found in the bulk of the container. In particular, swirls can be identified in time averaged velocity fields, the flow and the rods are aligned, and signatures of incipient clustering can be observed. To augment these results and extend them toward larger system sizes, we perform numerical simulations using a discrete-element molecular dynamics algorithm. In particular, we show the importance of particle aspect ratio and driving fluctuations on the observed pattern formation.

About $10^{3}$ polar rods were built using white hollow nylon cylinders of length $l=9.5 \mathrm{~mm}$ and diameter $d=4.76$ $\mathrm{mm}$, so the aspect ratio of the rods $A_{r}$ was fixed at 2 (see Fig. 1(a), Inset). Solid steel cylinders of length $4.75 \mathrm{~mm}$ and diameter $2.5 \mathrm{~mm}$ were placed snugly in one end of the nylon tube, which resulted in the center of mass being displaced by $0.1 \mathrm{l}$ from the geometrical center of the rod. The total mass of the assembly was $2.20 \times 10^{-4} \mathrm{~kg}$. The steel inserts also made the corresponding ends to appear somewhat darker and were used to identify the "polarity" of the rods. The particles were placed on a flat anodized aluminum container of radius $R=30 d$. 

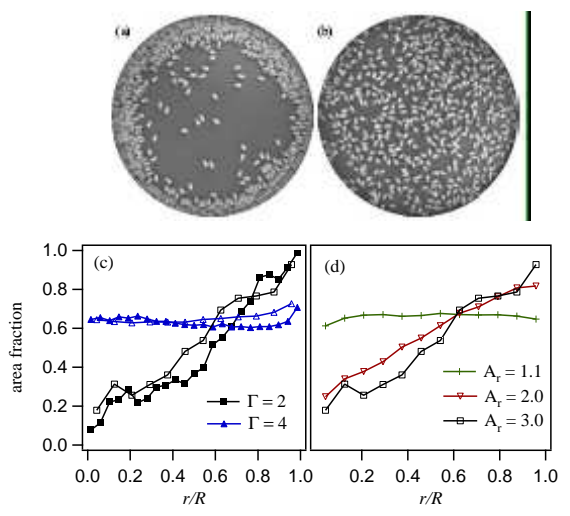

FIG. 2: (a) Rods migrate and aggregate at the boundaries of a container for modest excitations $(N=500, \Gamma=2)$. (b) Aggregation reduces and a homogeneous distribution is observed as excitation is increased. $(N=500, \Gamma=4)$. (c) Area fraction $\rho(r / R)$ as a function of distance $r$ from the center of the container with radius $R$ for $N=900$ averaged over 100 frames at 10 frames per second, open symbols: the results of numerical simulations for the same system parameters; (d) Simulations show decrease of clustering as $A_{r}$ is reduced $(\Gamma=2)$.

The container was vertically vibrated using an electromagnetic shaker with sinusoidal waveform at frequency $75 \mathrm{~Hz}$ and varied driving acceleration $\Gamma$ (scaled by the gravity acceleration) from 0 to 5 . A digital camera with the spatial resolution of $1000 \times 1000$ pixels was used to image the motion of rods inside the container.

First, we studied the motion of a single rod bouncing on the vibrated plate away from side walls. For $\Gamma>1.5$, the rod shows a robust net motion in the direction of the lighter end of the rod while taking some apparently random steps in the other directions as well. A movie of the typical motion is contained in the Supplementary material. By cross-correlating the intensity distribution of the image of the rod with a mask, we automated finding the position and the orientation (measured by the angle $\phi$ to a fixed axis) of the rod in each frame. By measuring the change in position over time interval $\tau$, the magnitude of the rod velocity $v$, and its direction $\theta$ with respect to a fixed reference were obtained. The probability distribution functions (PDF) for the displacement parallel to the $\operatorname{rod} v \tau \cos (\theta-\phi)$ and perpendicular to the $\operatorname{rod}$ $v \tau \sin (\theta-\phi)$ are plotted in Fig. 1 $(\mathrm{a}, \mathrm{b})$ with $3 \times 10^{5}$ sets of measurements. While the PDF in the perpendicular direction are centered at zero, the broader PDF in the parallel direction are clearly shifted from zero, and this shift grows as $\tau$ is increased. The mean and the rms velocity increase with $\Gamma$ in our system (see Supplementary materials.) By imaging from the side, we find that rods undergo short collisions with the bottom of the container once every few cycles at random phases of the cycle (see Supplementary materials.)

The physical mechanism for the observed directed motion in our polar rods can be understood by extend- ing the arguments developed for symmetric rods and dimers [17, 20]. During a typical collision of a particle with a horizontal plate, a large but short impulse of frictional force at the contact point causes horizontal particle displacement after the collision. When a symmetrical (apolar) rod bounces symmetrically on a vibrated plate, the net displacement after many collisions is absent, but for an asymmetric mode of vibration (as in Ref. 20]) or for an asymmetric particle (as in the present study), there is a non-zero net horizontal motion. In the case of polar rods, since the center of mass is displaced from the geometrical center, the heavy end collides more often with the plate, and the rod on average travels in the direction of the light end. It can be shown that the average horizontal velocity of the rod translation is proportional to the amplitude of the vertical speed of the container, and indeed we observe that the mean velocity increases with $\Gamma$.

The collective motion of polar rods was studied by placing the rods randomly initially inside the container and then vibrating with various $\Gamma$. (Movies included in Supplementary materials.) For low $\Gamma \sim 2$, rods were observed to migrate to the boundary of the container and aggregate in about 30 seconds. An example is shown in Fig. 2(a). Not all rods aggregate at the boundaries, as some rods gradually rotate and escape from the dense cluster at the boundary back into the middle of the container. As $\Gamma$ is increased, so do fluctuations, and the aggregation at the boundaries becomes less and less pronounced. Although spatio-temporal density inhomogeneities persist, the time-averaged number density of the polar rods appear more or less uniform across the cavity for $\Gamma>3$ (see Fig. 2(b)).

Next, we performed "molecular dynamics" simulations of polar rod motion and interaction. We did not simulate the details of the vibrational transport of bouncing rods, but instead assume that the rods were confined to a horizontal plane. A force acts on each rod along its (horizontal) axis in the direction of the lighter end. This force was assumed to be random, with a mean $F$ and variance $V$. In addition to the driving force, we assumed that rods experience velocity-dependent friction with the substrate and inelastic collisions with other rods. $F$ and $V$ were tuned so the displacement distribution for a single rod fits the experimental data for a given $\Gamma$. In the numerics, the rods had a form of spherocylinders, which helped in modeling contact forces. The interaction forces among rods were calculated via the interaction between viscoelastic virtual spheres of diameter $d$ centered at the closest points between the axes of the spherocylinders [17]. Normal forces were computed using Hertzian spring-dashpot model, and dynamic Coulomb friction was assumed for tangential forces. We did not add random forcing in the direction perpendicular to the axis of the rod, so the rods could only change their direction by colliding with the walls or other rods. 

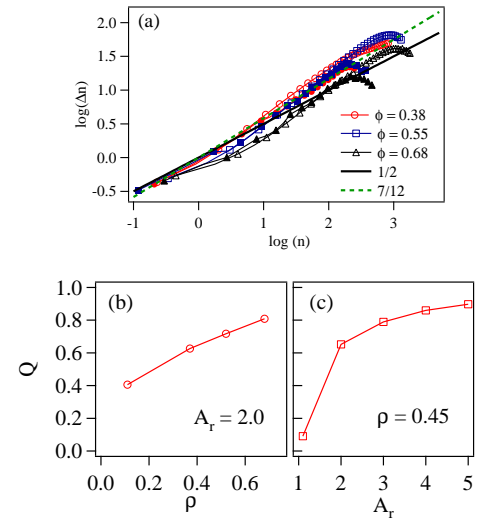

FIG. 3: (a) The standard deviation of the number of rods $\Delta n$ versus mean number of rods $n$ inside a circular area at the center of the container $(\Gamma=3)$. Open symbols correspond to the simulations for a larger system size $R_{L}=2.5 R_{S}$ but the same density. We consistently observe that $\Delta n$ grows faster than $\sqrt{n}$, which is a signature of a clustering regime. Local orientational order $Q$ for nearest neighbors from simulation data as a function of (b) density, and (c) aspect ratio.

We first performed simulations which matched the experiment both in terms of number of rods and the system geometry. We used aspect ratio $A_{r}=2$ and imposed elastic boundary conditions on a circle of radius $R_{S}=34.2 d$ 22]. For $F=0.25, V=0.16$ which correspond to $\Gamma=2$, we find that rods tend to aggregate at the boundary in agreement with experiment. As $F$ and $V$ are increased, the aggregation at the boundaries diminishes, also in accord with the experimental observations (see numerical movies in the Supplementary material). To illustrate and compare the aggregation of rods in the experiments and simulations, we plot the projected rod area fraction $\rho$ as a function of distance from the center $r$ in Fig. 2(c).

Clustering at the walls is not simply the consequence of inelastic collisions. Indeed, aggregation doesn't occur at the boundary for small $A_{r}=1.1$ (Fig. 2(d)), which indicates that particle shape affects aggregation. When fluctuations are small (at small $\Gamma$ ), rods have a much lower probability of turning around and leaving the wall than spherical particles, and so they are trapped near the wall for a long time.

In order to characterize the density fluctuations inside the container, we obtain the standard deviation $\Delta n$ and the mean $n$ of the number of rods in areas of different sizes by averaging over many realizations (see Fig. 3(a)). The distributions were obtained by averaging over 1500 frames after the system reached a statistically stationary regime, and we restricted the area of measurements to $r / R<0.7$ to minimize boundary effects. The data is systematically higher than $\sqrt{n}$. In fact, they are better described by the slope $7 / 12$ which is predicted by the dynamic XY model [7] in the nematic state. At very high values of $n$ the standard deviation drops down, as should be expected since the number of rods becomes comparable with the total number of rods in the container. In the numerical simulations which are also plotted in Fig. 3(a), we examine a larger system with $R_{L}=2.5 R_{s}$ and almost an order of magnitude greater number of rods. The deviations from $\sqrt{n}$ are also clearly present in this larger system. It is interesting to contrast these results with "giant" $(\Delta n \sim n)$ fluctuations reported for apolar rods [11, 16]. Although rods in our system have apolar shape, they have mass anisotropy which renders them polar and self-propelled under external vibration. This polarity appears to destroy the emergence of giant density fluctuations in agreement with earlier theoretical work [7].

Although global orientational order is clearly absent in our system, there is a strong evidence of the local orientational order at sufficiently high density of rods. We can characterize this ordering by computing a local orientational order parameter $Q$ which we define as $Q=$ $\langle\cos 2 \Theta\rangle$ where $\Theta$ is the angle between directors of a $\operatorname{rod}$ and its nearest neighbor and brackets indicate averaging over all the rods in the container and time (see Fig. 3bc). Parameter $Q$ is similar to the local orientational order parameter $S$ introduced in [6] and shows significant local orientational order present in our system at high enough $\rho$ and $A_{r}$.

Collective motion of rods in the container is masked by the strong random fluctuations, especially at high $\Gamma$. To reduce these fluctuations, we divided the field of view into $2 d \times 2 d$ boxes and averaged the velocity field over the box area and over a $\tau=5$ second time interval. An example of the obtained velocity field is shown in Fig. [4(a). This procedure reveals a number of streams and swirls. Numerical simulations for similar parameters also show swirl-like structures [see Fig. \&(b)]. The coherent structures become more pronounced when the system size is increased [see Fig. 4(c)]. These structures are reminiscent of swirls obtained with apolar particles driven by the substrate [12, 15]. However this is not entirely unexpected since the tensor order parameter for apolar particles in 2D has only two independent components and the corresponding order parameter equation can be reduced to a pseudo-vector form [15] which is similar to a vector order parameter equation for polar systems [10, 19, 21].

To quantify the structure of swirls, we plot in Fig. 4(d) the spatial velocity correlation function $C(r)=\left\langle\mathbf{v}_{1}\right.$. $\left.\mathbf{v}_{\mathbf{2}}\right\rangle /\left\langle\left|\mathbf{v}_{1}\right|\left|\mathbf{v}_{2}\right|\right\rangle$ for a rod with velocity $\mathbf{v}_{1}$ and a rod with velocity $\mathbf{v}_{2}$ separated by distance $r$. The correlations decay over a distance of a few rod lengths which confirms the lack of the long-range order in the system. However, the structure of the velocity field is strongly correlated with the orientation of the rods. We computed the distribution of the angle between the direction of the velocity field $\theta$ in and the mean orientation within a $(2 d \times 2 d)$ box both in experiment and numerical simulations, see 

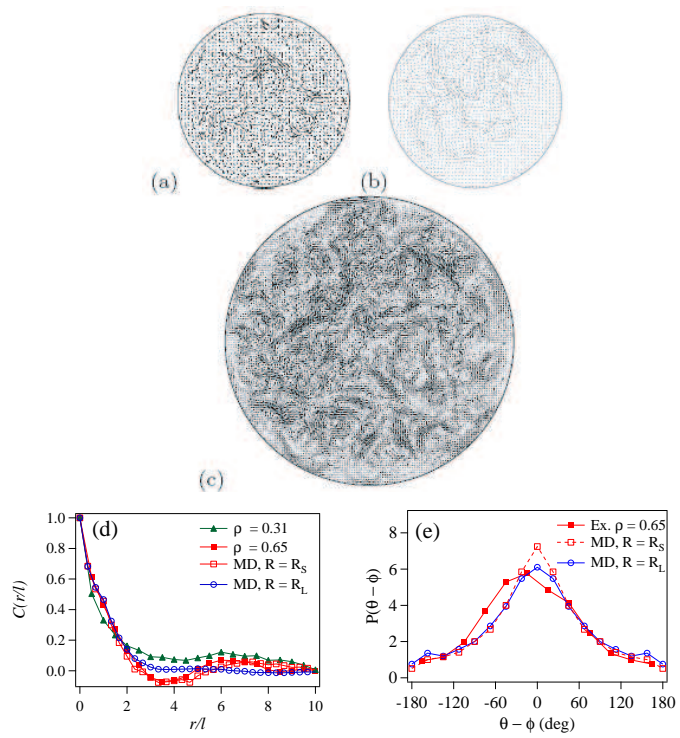

FIG. 4: Swirling in time averaged velocity obtained by computing particle displacements after $\tau=5 \mathrm{~s}$ : (a) experiment $(\Gamma=3 N=900)$, (b) numerics $\left(F_{0}=1.0, \rho=0.68\right),(\mathrm{c})$ Example of swirls observed in a larger numerical system $\left(N=5500, R_{L}=2.5 R_{S}\right)$. (d) Spatial velocity correlation function $C(r)$ as a function of distance between two rods $(\Gamma=3, \rho=0.31, \rho=0.65)$; results of simulations are shown for small $\left(R_{S}=34.2 d\right)$ and large $R_{L}=2.5 R_{S}$ system sizes and for the same density $\rho=0.68$. (e) The distribution of the angle between rod orientation and its velocity.

Fig. 4(e). As seen in Fig. 4(e), there is a significant maximum of this distribution at angle 0 , which indicates that rods predominantly move along their axes.

In summary, we have studied the collective dynamics of "self-propelled" polar rods with experiments and numerical simulations. The phenomenology differs qualitatively from that of collective motion of both point-like self-propelled particles [6] (which show no tendency to aggregate near the walls and get involved in system-size collective motion) and apolar rods [11, 12, 16] (which exhibit giant density fluctuations). We observe aggregation of rods at the boundaries because of the inability of rods to turn around and escape for high enough density under low noise conditions. As vibration strength and thus noise is increased, the aggregation reduces and a uniformly distributed state displaying local orientational order and swirls are observed. We observe greater than $\sqrt{n}$ density fluctuations which are in a qualitative agreement with model [7], but this agreement should not be over-emphasized since the model is directly applicable to a nematic regime. In our opinion, the observed deviation from the $\sqrt{n}$ regime for interacting polar rods is not accounted for by existing models and deserves further study. In conclusion, our findings elucidate an important and interesting interplay between the shape and the di- rected motion in realistic self-propelled rods which affects the phenomenology of their collective dynamics.

The work was supported by the National Science Foundation under Grant No. DMR-0605664 and the U.S. Department of Energy under Grant No. DE-FG0204ER46135.

[1] C.W. Reynolds, Computer Graphics 21, 25 (1987).

[2] J. Toner, Y. Tu, and S. Ramaswamy, Ann. Phys. (Berlin) 318, 170 (2005).

[3] E.O. Budrene and H.C. Berg, Nature (London) 349630 (1991); 376, 49 (1995); E.Ben-Jacob, et al. Nature 373 566 (1995).

[4] C. Dombrowski, et al. Phys. Rev. Lett. 93, 098103 (2004); A. Sokolov, et al., Phys. Rev. Lett. 98, 158102 (2007).

[5] T. Vicsek, et al., Phys. Rev. Lett. 75, 1226 (1995).

[6] G. Grégoire and H. Chaté, Phys. Rev. Lett. 92, 025702 (2004); H. Chaté, F. Ginelli, and R. Montagne, Phys. Rev. Lett. 96, 180602 (2006); M. Aldana, et al., Phys. Rev. Lett. 98, 095702 (2007).

[7] J. Toner and Y. Tu, Phys. Rev. Lett. 75, 4326 (1995); J. Toner, personal communication.

[8] E. Bertin, M. Droz, G. Grégoire, Phys. Rev. E 74, 022101 (2006).

[9] T. B. Liverpool and M.C. Marchetti, Phys. Rev. Lett. 90, 138102 (2003); 97, 268101 (2006); K. Kruse, et al., Phys. Rev. Lett. 92, 078101 (2004).

[10] I.S. Aranson and L.S. Tsimring. Phys. Rev. E 71, 050901 (2005); Phys. Rev. E 74, 031915 (2006).

[11] S. Ramaswamy, R. A. Simha, and J. Toner, Europhys. Lett. 62, 196 (2003); S. Mishra and S. Ramaswamy, Phys. Rev. Lett. 97, 090602 (2006).

[12] V. Narayan, N. Menon, and S. Ramaswamy, J. Stat. Mech.: Theory Exp. P01005 (2006).

[13] F. Peruani, A. Deutsch, and M. Bär, Phys. Rev. E 74, 030904(R) (2006).

[14] J. Galanis, et al., Phys. Rev. Lett. 96, 028002 (2006).

[15] I.S. Aranson, D. Volfson, and L.S. Tsimring. Phys. Rev. E 75, 051301 (2007)

[16] V. Narayan, S. Ramaswamy, and N. Menon, Science, 317, 105 (2007).

[17] D. Volfson, A. Kudrolli, and L. Tsimring, Phys. Rev. E 70, 051312(2004).

[18] D.L. Blair, T. Neicu, and A. Kudrolli, Phys. Rev. E 67, 031303 (2003).

[19] I.S. Aranson and L.S.Tsimring, Phys. Rev. E, 67, 021305 (2003).

[20] S. Dorbolo, D. Volfson, L. Tsimring, and A. Kudrolli, Phys. Rev. Lett. 95, 044101 (2005).

[21] I.S. Aranson, et al. Phys. Rev. E 75, 040901 (2007).

[22] $R$ in the simulations was chosen slightly bigger than in experiments to account for the different shapes of particles in order to match the areal density of rods.

Supplementary materials:

http://physics.clarku.edu/ akudrolli/polarods 